\title{
Dilation of Newton Polytope and $p$-adic Estimate
}

\author{
Wei Cao
}

Received: 19 September 2009 / Accepted: 17 January 2010 / Published online: 9 February 2010

(C) Springer Science+Business Media, LLC 2010

\begin{abstract}
Let $f(X)$ be a polynomial in $n$ variables over the finite field $\mathbb{F}_{q}$. Its Newton polytope $\Delta(f)$ is the convex closure in $\mathbb{R}^{n}$ of the origin and the exponent vectors (viewed as points in $\mathbb{R}^{n}$ ) of monomials in $f(X)$. The minimal dilation of $\Delta(f)$ such that it contains at least one lattice point of $\mathbb{Z}_{>0}^{n}$ plays a vital pole in the $p$-adic estimate of the number of zeros of $f(X)$ in $\mathbb{F}_{q}$. Using this fact, we obtain several tight and computational bounds for the dilation which unify and improve a number of previous results in this direction.
\end{abstract}

Keywords Finite field $\cdot$ Newton polytope $\cdot$ Lattice point $\cdot p$-adic estimate

\section{Introduction}

The theory of convex polytopes has many applications across mathematics and computer science (e.g., see [6, 9-11]). Given a multivariate polynomial one may associate with it an integral polytope called its Newton polytope. Let $K$ be an arbitrary field and $K^{*}=K-\{0\}$. Suppose that $f\left(x_{1}, \ldots, x_{n}\right) \in K\left[x_{1}, \ldots, x_{n}\right]$ is a nonconstant polynomial that is written in sparse representation as a sum of $m$ nonzero monomials

$$
f\left(x_{1}, \ldots, x_{n}\right)=\sum_{j=1}^{m} a_{j} x^{D_{j}}, \quad a_{j} \in K^{*},
$$

where

$$
D_{j}=\left(d_{1 j}, \ldots, d_{n j}\right) \in \mathbb{Z}_{\geq 0}^{n}, \quad x^{D_{j}}=x_{1}^{d_{1 j}} \cdots x_{n}^{d_{n j}} .
$$

W. Cao $(\bowtie)$

Department of Mathematics, Shanghai Jiaotong University, Shanghai 200240, China e-mail: caowei433100@vip.sina.com 
The set $\operatorname{Supp}(f):=\left\{D_{1}, \ldots, D_{m}\right\}$ is called the support of $f$. The Newton polytope $\Delta(f)$ is defined to be the convex closure in $\mathbb{R}^{n}$ of the set $\operatorname{Supp}(f) \cup O$ where $O$ denotes the origin of $\mathbb{R}^{n}$; in our notation, $\Delta(f)=\operatorname{conv}(\operatorname{Supp}(f) \cup O)$.

Remark 1 The Newton polytope $\Delta(f)$ has also been defined to be the convex closure of the set $\operatorname{Supp}(f)$ by some authors in the literature. However, for the case $O \in$ $\operatorname{conv}(\operatorname{Supp}(f))$ (e.g., $f$ has a nonzero constant), these two definitions are coincide with each other.

The Newton polytope provides a geometric approach to studying polynomials. For example, according to the BKK bound (named after Bernshtein, Khovanskii and Kouchnirenko; see [3,14-16]), the number of common zeros in $\left(\mathbb{C}^{*}\right)^{n}$ of $n$ generic polynomials is bounded by the volume of their Newton polytopes. The decomposition of the Newton polytope, in the sense of the Minkowski sum, has a close relation to the reducibility of the associated polynomial, which was observed by Ostrowski [19] and developed by Gao and Lauder [7, 8] and others. For the polynomial defined over finite fields, Adolphson and Sperber [1] discovered that the dilation of its Newton polytope can be used to estimate the $p$-divisibility of the number of zeros of the polynomial. This paper will be devoted to this topic.

Let $\mathbb{F}_{q}$ be the finite field of $q$ elements with characteristic $p$. Suppose that $f\left(x_{1}, \ldots, x_{n}\right)$ is a polynomial in $n$ variables of degree $d$ over $\mathbb{F}_{q}$. Let $N(f)$ denote the number of zeros of $f$ in $\mathbb{F}_{q}$ and $\operatorname{ord}_{p}\left(\operatorname{resp} . \operatorname{ord}_{q}\right)$ the $p$-adic additive valuation normalized such that $\operatorname{ord}_{p} p=1\left(\operatorname{resp} . \operatorname{ord}_{q} q=1\right)$. One of the problems of interest is the estimate of its $p$-divisibility. The classical Chevalley-Warning theorem asserts $\operatorname{ord}_{p} N(f) \geq 1$ if $n>d$. Let $\lceil x\rceil$ denote the least integer $\geq x$ for $x \in \mathbb{R}$, Ax showed that

\section{Theorem 1 ([2])}

$$
\operatorname{ord}_{q} N(f) \geq\left\lceil\frac{n}{d}\right\rceil-1
$$

Ax's result was extended by Katz [13] to a system of equations by using Dwork's $p$-adic theory. Wan [21] provided an elementary proof to the Ax-Katz theorem. The simplest proof of the $\mathrm{Ax}-\mathrm{Katz}$ theorem and its extension to character sums were given in [22]. A reduction of the $\mathrm{Ax}-\mathrm{Katz}$ theorem for a system of equations to Ax's theorem for a single equation has been found by Hou [12]. O. Moreno and C.J. Moreno [18] improved the Ax-Katz theorem in terms of $p$-weight degree in certain special cases. Recently, Cao and Sun $[4,5]$ obtained some new results improving Chevalley-Warning-Ax-Katz-type estimate for the general diagonal polynomials over finite fields.

In this paper, we will extend and improve Ax's theorem by using the dilation of Newton polytope. Instead of considering all the variables, we will focus on those variables with low degree. Put $\Omega=\{1,2, \ldots, n\}$. For any nonempty subset $A \subset \Omega$, let $f_{A}$ be the polynomial obtained from $f$ by letting $x_{i}=1$ for $i \in \Omega-A$. As usual, let $|A|$ denote the cardinality of $A$. Clearly, $f_{\Omega}=f$. Our result can be stated as 
Theorem 2 (Main Theorem) For any nonempty subset $A \subset \Omega$, we have

$$
\operatorname{ord}_{q} N(f) \geq\left\lceil\frac{|A|}{\operatorname{deg} f_{A}}\right\rceil-1 .
$$

Corollary 1 If $|A|>\operatorname{deg} f_{A}$ for some nonempty subset $A \subset \Omega$, then $N(f)>0$ and $q \mid N(f)$.

Example 1 Let $f\left(x_{1}, x_{2}, x_{3}, x_{4}\right)=x_{1} x_{3}^{4}+x_{2} x_{4}^{5}$. Since deg $f=6$ and $n=4$, one gets the trivial estimate $q^{0} \mid N(f)$ by Ax's theorem. Now take $A=\{1,2\}$, then $f_{A}=$ $x_{1}+x_{2}$, and one gets $q \mid N(f)$ by our main theorem.

As one of the reviewers pointed out, the Newton polytope of a multivariate polynomial has an internal structure which is only visible when one considers the complete family of polytopes associated to all the possible polynomials that arise by deleting one or more terms in the polynomial. Theorem 2 is an inductive argument that justifies this claim. The remainder of the paper is organized in the following way. The proof of Theorem 2 will be given in Sect. 2, before which the result of Adolphson and Sperber will be briefly introduced. In Sect. 3, we will give several bounds in special cases which unify and improve a number of previous results in this direction.

\section{Adolphson-Sperber's Result and Proof of Main Theorem}

By using the tool of Newton polytope and Dwork's $p$-adic theory, Adolphson and Sperber [1] generalized Ax's theorem to the exponential sums (for the knowledge of exponential sums, refer to Lidl and Niederreiter [17]). Let $\zeta_{p}$ be a fixed primitive $p$-th root of unity in the complex numbers. For a given polynomial $f\left(x_{1}, \ldots, x_{n}\right) \in$ $\mathbb{F}_{q}\left[x_{1}, \ldots, x_{n}\right]$ of the form as in (1), we form the exponential sum

$$
S(f)=\sum_{x_{1}, \ldots, x_{n} \in \mathbb{F}_{q}} \zeta_{p}^{\operatorname{Tr}_{\mathbb{F}_{q} / \mathbb{F} p}\left(f\left(x_{1}, \ldots, x_{n}\right)\right)}
$$

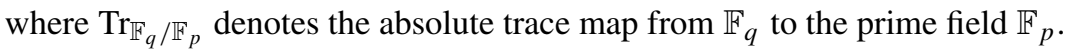

Let $\Delta(f)$ be the Newton polytope of $f$ as defined at the beginning of Sect. 1 . Let $\omega(f)$ be the smallest positive rational number such that the dilation $\omega(f) \Delta(f)$ contains a lattice point of $\mathbb{Z}_{>0}^{n}$. Adolphson and Sperber proved

Theorem 3 ([1, Theorem 1.2]) ord $_{q} S(f) \geq \omega(f)$.

Let $x_{0} f$ be the new polynomial by adding a further variable $x_{0}$ to the original polynomial $f$. It is easy to verify that the relation between $N(f)$ and $S\left(x_{0} f\right)$ is

$$
q N(f)=S\left(x_{0} f\right) \quad \Longrightarrow \quad \operatorname{ord}_{q} N(f)=\operatorname{ord}_{q} S\left(x_{0} f\right)-1 .
$$

By Theorem 3, (3) and the fact that $N(f) \in \mathbb{Z}_{\geq 0}$, we have 
Corollary $2 \operatorname{ord}_{q} N(f) \geq\left\lceil\omega\left(x_{0} f\right)\right\rceil-1$.

For a concrete polynomial whose Newton polytope is known in advance, the exact value of $\omega(f)$ (or $\omega\left(x_{0} f\right)$ ) can be calculated by standard arguments in linear programming. However, we still want to find a tight and computational estimate for $\omega(f)$ (or $\omega\left(x_{0} f\right)$ ) of the simple expression like (2) in the general case. This is just what Theorem 2 tells.

Recall a basic fact from convex geometry: all points in $\Delta(f)$ can be written in the form

$$
P=\sum_{i=0}^{m} \beta_{i} D_{i} \quad \text { where } \beta_{i} \geq 0, \quad \text { and } \quad \sum_{i=0}^{m} \beta_{i}=1 .
$$

Here we set $D_{0}:=O=(0, \ldots, 0)$ for convenience.

\section{Lemma 1}

$$
\omega(f)=\min \left\{\sum_{i=0}^{m} \alpha_{i} \mid \sum_{i=0}^{m} \alpha_{i} D_{i} \in \mathbb{Z}_{>0}^{n}, \alpha_{i} \in \mathbb{Q}_{\geq 0}\right\}
$$

Proof Assume that the point $P=\sum_{i=0}^{m} \alpha_{i} D_{i}$ with $\alpha_{i} \in \mathbb{Q}_{\geq 0}$ is a lattice point of $\mathbb{Z}_{>0}^{n}$. Let $\alpha=\sum_{i=1}^{m} \alpha_{i}$. We claim $\alpha>0$; Otherwise, $P=D_{0} \notin \mathbb{Z}_{>0}^{n}$, a contradiction. Now consider the new point $P^{\prime}=\sum_{i=0}^{m} \frac{\alpha_{i}}{\alpha} D_{i}$. Since $\sum_{i=0}^{m} \frac{\alpha_{i}}{\alpha}=1$, we have $P^{\prime} \in \Delta(f)$. Clearly, $\alpha P^{\prime}=P \in \mathbb{Z}_{>0}^{n}$ by our assumption. So we get $\omega(f) \leq \alpha$. The result follows from the minimality of $\omega(f)$.

We are now in a position to prove the main theorem of this paper.

Proof of Theorem 2 Without loss of generality, assume $A=\{1,2, \ldots, k\}$ with $k \leq n$. Thus

$$
f_{A}=a_{1} x_{1}^{d_{11}} \cdots x_{k}^{d_{k 1}}+\cdots+a_{m} x_{1}^{d_{1 m}} \cdots x_{k}^{d_{k m}} .
$$

Put $d_{j}:=\sum_{i=1}^{k} d_{i j}$ for $j=1, \ldots, m$. Then $\operatorname{deg} f_{A}=\max \left\{d_{j} \mid j=1, \ldots, m\right\}$. Assume that the point $P=\sum_{i=0}^{m} \alpha_{i} D_{i}$ with $\alpha_{i} \in \mathbb{Q}_{\geq 0}$ is a lattice point of $\mathbb{Z}_{>0}^{n}$, then we obtain the following system:

$$
\left\{\begin{array}{l}
\alpha_{1} d_{11}+\cdots+\alpha_{m} d_{1 m}=\lambda_{1}, \\
\vdots \\
\alpha_{1} d_{k 1}+\cdots+\alpha_{m} d_{k m}=\lambda_{k},
\end{array}\right.
$$

with $\lambda_{i} \in \mathbb{Z}_{>0}$ for $i=1, \ldots, k$. Summing up the above equalities yields

$$
\alpha_{1} d_{1}+\cdots+\alpha_{m} d_{m}=\lambda_{1}+\cdots+\lambda_{k} .
$$

Dividing the both sides of (4) by $\operatorname{deg} f_{A}$, we get

$$
\sum_{i=1}^{m} \alpha_{i} \geq \alpha_{1} \frac{d_{1}}{\operatorname{deg} f_{A}}+\cdots+\alpha_{m} \frac{d_{m}}{\operatorname{deg} f_{A}}=\frac{\lambda_{1}+\cdots+\lambda_{k}}{\operatorname{deg} f_{A}} \geq \frac{k}{\operatorname{deg} f_{A}} .
$$


Here we use the fact that $\operatorname{deg} f_{A} \geq d_{i}$ in the first inequality and the fact that $\lambda_{i} \geq 1$ in the last inequality. By Lemma 1 and (5), we have

$$
\omega\left(x_{0} f\right) \geq \frac{|A|}{\operatorname{deg} f_{A}} .
$$

The result follows from Corollary 2 and (6).

Remark 2 Clearly, there are $2^{n}-1$ choices for $A$. The key problem is how to find a suitable set $A$ which corresponds to the maximal value of $|A| / \operatorname{deg} f_{A}$. We have given an example in Example 1. In fact, it is just a particular case of Theorem 6 below.

\section{Further Improvement}

Wan [20], Cao and Sun [4, 5] have respectively investigated the polynomials of special form over finite fields and obtained the $p$-adic estimate for their numbers of zeros. It can be easily seen that their estimates are tighter than Ax's estimate. In this section, we will show that their results can be deduced or improved by using our technique.

Theorem 4 ([20]) Let $f(X)$ be a diagonal polynomial of the form

$$
f(X)=a_{1} x_{1}^{d_{1}}+a_{2} x_{2}^{d_{2}}+\cdots+a_{n} x_{n}^{d_{n}}+c,
$$

where $a_{i} \in \mathbb{F}_{q}^{*}, c \in \mathbb{F}_{q}$ and $d_{i} \in \mathbb{Z}_{>0}$. Then we have

$$
\operatorname{ord}_{q} N(f) \geq\left\lceil\frac{1}{d_{1}}+\frac{1}{d_{2}}+\cdots+\frac{1}{d_{n}}\right\rceil-1 .
$$

Note that in (7) one has $d:=\operatorname{deg} f=\max \left\{d_{1}, \ldots, d_{n}\right\}$ which implies that $\frac{1}{d_{1}}+$ $\frac{1}{d_{2}}+\cdots+\frac{1}{d_{n}} \geq \frac{n}{d}$. This shows that Wan's theorem is stronger than Ax's theorem for diagonal cases. form

Cao and Sun $[4,5]$ have studied the so-called general diagonal polynomial of the

$$
f=\sum_{i=1}^{r} a_{i} x_{i 1}^{d_{i 1}} x_{i 2}^{d_{i 2}} \cdots x_{i n_{i}}^{d_{i n_{i}}}+c
$$

where $a_{i} \in \mathbb{F}_{q}^{*}, c \in \mathbb{F}_{q}, d_{i j} \in \mathbb{Z}_{\geq 0}$ and $x_{i j}$ 's are $n\left(=\sum_{i=1}^{r} n_{i}\right)$ distinct variables. Set $d_{i}=\operatorname{gcd}\left(d_{i 1}, \ldots, d_{i n_{i}}, q-1\right)$ for $i=1, \ldots, r$, and define the new polynomial

$$
\widehat{f}=\sum_{i=1}^{r} a_{i}\left(x_{i 1} x_{i 2} \cdots x_{i n_{i}}\right)^{d_{i}}+c .
$$

Theorem 5 ([5]) With the notations as above, we have

$$
N(f)=N(\widehat{f}) \quad \Longrightarrow \quad \operatorname{ord}_{q} N(f) \geq\left\lceil\frac{n}{\operatorname{deg} \widehat{f}}\right\rceil-1 .
$$


Since $\operatorname{deg} \widehat{f} \leq \operatorname{deg} f$, Cao-Sun's result may also improve Ax's theorem. We first show that Theorem 4 can be deduced by the following theorem.

Theorem 6 Let $f(X, Y)$ be a polynomial of the form

$$
f(X, Y)=x_{1}^{d_{1}} g_{1}(Y)+\cdots+x_{n}^{d_{n}} g_{n}(Y)+c,
$$

where $g_{i}(Y) \in \mathbb{F}_{q}\left[y_{1}, \ldots, y_{m}\right]$ are polynomials, $c \in \mathbb{F}_{q}$ and $d_{i} \in \mathbb{Z}_{>0}$. Then

$$
\operatorname{ord}_{q} N(f) \geq\left\lceil\frac{1}{d_{1}}+\frac{1}{d_{2}}+\cdots+\frac{1}{d_{n}}\right\rceil-1 .
$$

Proof Consider the polynomial

$$
f(X)=x_{1}^{d_{1}}+x_{2}^{d_{2}}+\cdots+x_{n}^{d_{n}} .
$$

Applying Lemma 1 to (10), one has

$$
\alpha_{i} d_{i}=\lambda_{i} \geq 1 \quad \Longrightarrow \quad \alpha_{i}=\frac{\lambda_{i}}{d_{i}} \geq \frac{1}{d_{i}} \quad \Longrightarrow \quad \sum_{i=1}^{n} \alpha_{i} \geq \sum_{i=1}^{n} \frac{1}{d_{i}}
$$

The result follows from (11) and Corollary 2.

As one of the reviewers pointed out, Theorem 6 is also an immediate consequence of Theorem 4. For a given $y=\left(y_{1}, \ldots, y_{m}\right) \in \mathbb{F}_{q}^{m}$, let $N(f(X, y))$ denote the number of zeros of $f(X, y)$ in $\mathbb{F}_{q}$ and set $h:=\left\lceil\frac{1}{d_{1}}+\frac{1}{d_{2}}+\cdots+\frac{1}{d_{n}}\right\rceil-1$. Then $q^{h} \mid N(f(X, y))$ for all $y=\left(y_{1}, \ldots, y_{m}\right) \in \mathbb{F}_{q}^{m}$ by Theorem 4. Therefore one has $q^{h} \mid N(f)$.

Now we show that Theorem 5 can be generalized and improved by Theorem 6 .

Corollary 3 Let $f$ be a general diagonal polynomial of the form as in (8) and $\widehat{f}$ defined as in (9). Then we have

$$
\operatorname{ord}_{q} N(f) \geq\left\lceil\frac{1}{d_{1}}+\frac{1}{d_{2}}+\cdots+\frac{1}{d_{r}}\right\rceil-1 \geq\left\lceil\frac{n}{\operatorname{deg} \widehat{f}}\right\rceil-1 .
$$

The second inequality shows that it improves Theorem 5 and hence Ax's theorem.

Proof Consider the polynomial

$$
\widehat{\widehat{f}}=\sum_{i=1}^{r} a_{i} x_{i 1}^{d_{i}}
$$

The first inequality in (12) follows from the fact that $N(f)=N(\widehat{f})$ (see Theorem 5) and Theorem 6. Since $\operatorname{deg} \widehat{f}=\max \left\{n_{i} d_{i} \mid i=1, \ldots, r\right\}$ and $n=\sum_{i=1}^{r} n_{i}$, one gets

$$
\frac{n}{\operatorname{deg} \widehat{f}} \leq \frac{n_{1}}{n_{1} d_{1}}+\cdots+\frac{n_{r}}{n_{r} d_{r}} \leq \frac{1}{d_{1}}+\cdots+\frac{1}{d_{r}} .
$$

This yields the second inequality in (12). 
Remark 3 One sees that the Newton polytope in Theorem 6 is of special shape. The choice of $A$ in Theorem 2 as well as the tightness of estimate for $\omega(f)$ (or $\omega\left(x_{0} f\right)$ ) depends upon the shape of the associated Newton polytope. It may be an interesting problem to find a set $A$ such that the value of $|A| / \operatorname{deg} f_{A}$ is the greatest among all the choices of $A$.

Acknowledgements The author would like to thank the reviewers for their comments that helped improve the manuscript. This work was jointly supported by the National Natural Science Foundation of China (No. 10901101), China Postdoctoral Science Foundation (No. 20090450708) and Shanghai Postdoctoral Science Foundation (No. 09R21413900).

\section{References}

1. Adolphson, A., Sperber, S.: p-Adic estimates for exponential sums and the theorem of ChevalleyWarning. Ann. Sci. Ecole Norm. Super. 4th Ser. 20, 545-556 (1987)

2. Ax, J.: Zeros of polynomials over finite fields. Am. J. Math. 86, 255-261 (1964)

3. Bernshtein, D.N.: The number of roots of a system of equations. Funct. Anal. Appl. 9, 183-185 (1975)

4. Cao, W., Sun, Q.: A reduction for counting the number of solutions of general diagonal equation over finite fields. Finite Fields Their Appl. 12, 681-692 (2006)

5. Cao, W., Sun, Q.: Improvements upon the Chevalley-Warning-Ax-Katz-type estimate. J. Number Theory 122, 135-141 (2007)

6. Ewald, G.: Combinatorial Convexity and Algebraic Geometry. GTM, vol. 168. Springer, New York (1996)

7. Gao, S.: Absolute irreducibility of polynomials via Newton polytopes. J. Algebra 237, 501-520 (2001)

8. Gao, S., Lauder, A.G.: Decomposition of Polytopes and Polynomial. Discrete Comput. Geom. 26, 89-104 (2001)

9. Goodman, J.E., O’Rourke, J.: Handbook of Discrete and Computational Geometry. Elsevier, Amsterdam (1997)

10. Gruber, P.M., Wills, J.M.: Handbook of Convex Geometry, vols. A and B. Elsevier, Amsterdam (1993)

11. Grünbaum, B.: Convex Polytopes. GTM, vol. 221. Springer, New York (2003)

12. Hou, X.D.: A note on the proof of a theorem of Katz. Finite Fields Their Appl. 11, 316-319 (2005)

13. Katz, N.M.: On a theorem of Ax. Am. J. Math. 93, 485-499 (1971)

14. Khovanskii, A.G.: Newton polyhedra and the genus of complete intersections. Funct. Anal. Appl. 12, 38-46 (1978)

15. Kushnirenko, A.G.: Polyèdres de Newton et nombres de Milnor. Invent. Math. 32, 1-31 (1976)

16. Kushnirenko, A.G.: Newton polytopes and the Bezout theorem. Funct. Anal. Appl. 10, 233-235 (1976)

17. Lidl, R., Niederreiter, H.: Finite Fields. Encyclopedia of Mathematics and its Applications, vol. 20. Addison-Wesley, Reading (1983)

18. Moreno, O., Moreno, C.J.: Improvement of the Chevalley-Warning and the Ax-Katz theorem. Am. J. Math. 117, 241-244 (1995)

19. Ostrowski, A.M.: On multiplication and factorization of polynomials, I: Lexicographic ordering and extreme aggregates of terms. Aequ. Math. 13, 201-228 (1975)

20. Wan, D.: Zeros of diagonal equations over finite fields. Proc. Am. Math. Soc. 103, 1049-1052 (1988)

21. Wan, D.: An elementary proof of a Theorem of Katz. Am. J. Math. 111, 1-8 (1989)

22. Wan, D.: A Chevalley-Warning approach to $p$-adic estimates of character sums. Proc. AMS Math. Soc. 3, 45-54 (1995) 